\title{
Distribución de la longitud axial y factores relacionados en una población adulta de la Ciudad de México
}

\section{Distribution of axial length and related factors in an adult population of Mexico City}

\author{
M ${ }^{a}$ Soledad Barlatey*, Wilson Koga-Nakamura, M. ${ }^{a}$ Victoria Moreno-Londoño, Mariana Takane-Imay y \\ M. ${ }^{a}$ Cristina González-González
}

Servicio de Ecografía Ocular, Instituto de Oftalmología Fundación Conde de Valenciana, Ciudad de México, México

\section{Resumen}

Objetivo: Analizar la longitud axial, profundidad de cámara anterior, grosor del cristalino y profundidad de cámara vítrea en una población adulta de la ciudad de México y alrededores con diagnóstico de catarata. Métodos: Estudio retrospectivo y descriptivo. Se revisaron los expedientes de un período de 6 meses (de 1 de junio de 2013 a 31 de diciembre de 2013) de sujetos sometidos a un examen ecográfico como parte de su rutina prequirúrgica. Los parámetros biométricos estaban disponibles para 865 ojos después de aplicar los criterios de exclusión. Todas las mediciones fueron realizadas utilizando el biómetro ultrasónico Ocuscan RxP (Alcon Laboratories, Fort Worth, Texas, EE.UU). Resultados: El estudio incluyó 430 ojos. La longitud axial media fue de $23.44 \pm 1.71 \mathrm{~mm}$ (mediana: 23.15; rango: 20.48-35.05 mm; IC 95\%: 23.27-23.60). La profundidad de cámara anterior media fue de $3.12 \pm 0.46 \mathrm{~mm}$ (mediana: $3.13 \mathrm{~mm}$; rango: 1.77-4.54 mm; IC 95\%: 3.07-3.16). El grosor cristaliniano medio fue de $4.47 \pm 0.57 \mathrm{~mm}$ (mediana: $4.50 \mathrm{~mm}$; rango: 3.18-5.78; IC 95\%: 4.41-4.52). La profundidad de cámara vítrea media fue de $15.81 \pm 1.63 \mathrm{~mm}$ (mediana: $15.58 \mathrm{~mm}$; rango: 12.98-26.78; IC 95\%: 15.66-15.97). La edad media de los pacientes fue de $70.03 \pm 10.50$ años (mediana 70 años, rango 40-96 años). Conclusiones: En la población adulta mexicana de la Ciudad de México, particularmente en las mujeres, se encontró una menor longitud axial y profundidad de cámara anterior. Comparado con otros estudios, la longitud axial se encontró dentro de un rango promedio. La edad y el sexo estuvieron significativamente asociados con los demás parámetros evaluados en este estudio.

Palabras clave: Longitud axial. Profundidad de cámara anterior. Grosor cristaliniano. Profundidad de cámara vítrea.

\begin{abstract}
Background: To analyze the axial length, anterior chamber depth, lens thickness and vitreous chamber depth in an adult population from Mexico City and the surrounding area with a diagnosis of cataract. Methods: Retrospective and descriptive study. We reviewed the medical files of subjects undergoing an ultrasound examination as part of the pre-surgical routine evaluation during a period of 6 months (June 1, 2013-December 31,2013). Biometric parameters were available for 865 eyes after applying the exclusion criteria. All measurements were made using the Ocuscan ${ }^{\circledR} R x P$ ultrasonic biometer (Alcon Laboratories, Fort Worth, Texas, USA). Results: The study included 430 eyes. Mean axial length was $23.44 \pm 1.71 \mathrm{~mm}$ (median: 23.15, range: 20.48-35.05 mm, 23.27-23.60, 95\% confidence interval [Cl]). Mean anterior chamber depth was $3.12 \pm 0.46 \mathrm{~mm}$ (median:
\end{abstract}

\section{Correspondencia:}

*M. ${ }^{a}$ Soledad Barlatey

Chimalpopoca, 14

Col. Obrera, Del. Cuahutemoc

C.P. 06800, Ciudad de México, México

E-mail: sol_barlatey@ hotmail.com

Fecha de recepción: 29-01-2019

Fecha de aceptación: 13-06-2019

DOI: 10.24875/RMO.M19000083
Disponible en internet: 01-09-2019 Rev Mex Oftalmol. 2019;93(5):233-237

www.rmo.com.mx 0187-4519/@ 2019 Sociedad Mexicana de Oftalmología. Publicado por Permanyer México. Este es un artículo Open Access bajo la licencia CC BY-NC-ND (http://creativecommons.org/licenses/by-nc-nd/4.0/). 
Rev Mex Oftalmol. 2019;93

$3.13 \mathrm{~mm}$, range: $1.77-4.54 \mathrm{~mm}, 3.07-3.16,95 \% \mathrm{Cl}$ ). Mean lens thickness was $4.47 \pm 0.57 \mathrm{~mm}$ (median: $4.50 \mathrm{~mm}$, range: $3.18-5.78$, 4.41-4.52, 95\% Cl). Mean vitreous chamber depth was $15.81 \pm 1.63 \mathrm{~mm}$ (median: $15.58 \mathrm{~mm}$, range: 12.98-26.78, 15.66-15.97, $95 \% \mathrm{Cl}$ ). Mean age of the patients was $70.03 \pm 10.50$ years (median 70 years, range 40-96 years). Conclusions: In a Mexican adult population from Mexico City, particularly in women, a lower axial length and anterior chamber depth was observed. Compared to other studies, the axial length was found within an average range. Age and sex were significantly associated with the other parameters evaluated in this study.

Key words: Axial length. Anterior chamber depth. Lens thickness. Vitreous chamber depth.

\section{Introducción}

A finales del siglo XIX, el físico y matemático británico William Thompson Kelvin (1824-1907) decía que «lo que no se mide, no se puede mejorar». Tal es así que, la medida de la longitud axial (LA) es un parámetro oftálmico importante y necesario para el cálculo de la potencia del lente intraocular previo a la cirugía de catarata y la cirugía refractiva ${ }^{1}$, y es útil en el diagnóstico temprano de afecciones oculares como microftalmos y/o nanoftalmos, estafiloma ${ }^{2}$ y el riesgo de desprendimiento de retina ${ }^{3}$.

Varios estudios han demostrado la correlación entre la biometría ocular, especialmente la LA, con los errores refractivos. Hosny, et al. ${ }^{4}$ encontraron que el diámetro corneal, el equivalente esférico de la refracción y la profundidad de la cámara anterior (PCA) afectaban los parámetros de la LA. En un estudio realizado en la Universidad de Shangai por Wang, et al. ${ }^{5}$, en el que se midió la LA de 255 ojos, se encontró una LA de $23.63 \mathrm{~mm}( \pm 0.92)$ en individuos hipermétropes, $24.62 \mathrm{~mm}( \pm 0.38)$ en emétropes y $26.68 \mathrm{~mm}( \pm 0.75)$ en miopes. Dado que estos parámetros pueden estar influenciados por la raza, etnia y genética, sus diferencias entre las distintas poblaciones probablemente puedan explicar las variaciones en los errores de refracción.

Informes relacionados con la distribución de la biometría ocular en estudios poblacionales se han publicado desde algunos países asiáticos como Taiwán, Myanmar, Singapur y China ${ }^{6-9}$.

Una revisión de varios estudios usando diferentes técnicas de medición muestra que la medición de la LA del ojo normal adulto es de aproximadamente $23.60 \mathrm{~mm}^{10,11}$, tomada esta desde el ápice corneal hasta la membrana limitante interna (MLI). Sin embargo, la mayoría de los individuos se encuentran en un rango de 22.00 a $24.50 \mathrm{~mm}$.

Hoffer demostró que la PCA promedio en ojos fáquicos es de $3.24 \mathrm{~mm}( \pm 0,44 \mathrm{~mm})^{11}$ y concluyó, en un estudio sobre 7,500 ojos, que el grosor de un cristalino (GC) cataratoso es de $4.63 \mathrm{~mm}^{10}$.

\section{Objetivo}

En nuestro estudio se muestra la distribución de la LA y sus componentes, incluyendo la PCA, el GC y la profundidad de la cámara vítrea (PCV), en una población mexicana de 40 a 99 años con diagnóstico de catarata.

\section{Método}

Todas las adquisiciones fueron realizadas con el biómetro ultrasónico Ocuscan RxP (Alcon Laboratories, Fort Worth, Texas, EE.UU) durante el período de junio a diciembre de 2013. El biómetro ultrasónico mide córnea-MLI. Las mediciones fueron registradas en modo automático, con la técnica de inmersión, para evitar el aplanamiento corneal y obtener resultados comparables a la biometría óptica.

La LA es definida desde la cara anterior de la córnea hasta la MLI, la PCA es definida desde la cara anterior de la córnea (epitelio corneal) hasta la cara anterior del cristalino, el GC definido por sus dos caras, biconvexo, detrás del iris, y la PCV es definida desde la cara posterior del cristalino hasta la MLI.

Cada índice de biometría ocular se definió por su media y un intervalo de confianza (IC) del 95\% por edad y género, y el rango normal calculado como la media de \pm 2 desviaciones estándar. Los percentiles 5 , $10,25,50,75,90,95$ fueron determinados para mostrar la distribución de estas variables con más detalle.

Para evaluar las diferencias de distribución de lo normal, las distribuciones se trazaron en histogramas. Los coeficientes de correlación de Pearson se determinaron al examinar la correlación entre ambos ojos en términos de LA y sus componentes.

En este reporte solo los ojos fáquicos fueron incluidos para el análisis y cualquiera que tuviera una historia de cirugía ocular fue excluido. La correlación entre los ojos derechos e izquierdos fue alta en el caso de los índices de LA ( $r=0,997)$, PCA $(r=0,980), G C$ $(r=1,015)$ y PCV $(r=0,989)$, por lo tanto, solo se presentan aquí los resultados de los ojos derechos. 
Tabla 1. Distribución de la longitud axial (LA), profundidad de cámara anterior (PCA), grosor del cristalino (GC) y profundidad de cámara vítrea (PCV) con su media y $95 \%$ de intervalo de confianza de la media (IC) por edad y género

\begin{tabular}{|c|c|c|c|c|c|}
\hline Edad (años) & $N$ & LA (mm) Media (IC 95\%) & PCA (mm) Media (IC 95\%) & GC (mm) Media (IC 95\%) & PV (mm) Media (IC 95\%) \\
\hline $\begin{array}{c}\text { Hombres } \\
40-49\end{array}$ & 5 & $23.03(22.66-23.41)$ & $3.55(3.02-3.42)$ & $4.11(3.62-4.60)$ & $15.39(15.15-15.62)$ \\
$50-59$ & 19 & $23.99(23.66-24.32)$ & $3.47(3.27-3.66)$ & $4.15(3.93-4.37)$ & $16.36(16.04-16.68)$ \\
$60-69$ & 34 & $23.55(23.02-24.08)$ & $3.22(3.02-3.42)$ & $4.49(4.27-4.71)$ & $15.83(15.33-16.33)$ \\
$70-79$ & 40 & $23.89(23.48-24.30)$ & $3.25(3.10-3.40)$ & $4.39(4.20-4.58)$ & $16.23(15.84-16.62)$ \\
$80+$ & 31 & $23.67(23.39-23.95)$ & $3.17(3.01-3.33)$ & $4.60(4.40-4.80)$ & $15.89(15.61-16.17)$ \\
\hline Mujeres & & & & & \\
$40-49$ & 9 & $24.36(22.45-26.28)$ & $3.50(3.04-3.97)$ & $4.02(3.53-4.51)$ & $16.83(15.02-18.64)$ \\
$50-59$ & 43 & $23.56(22.81-24.81)$ & $3.16(3.02-3.31)$ & $4.21(4.04-4.38)$ & $16.16(15.45-16.86)$ \\
$60-69$ & 85 & $23.41(22.91-23.90)$ & $3.10(3.01-3.19)$ & $4.47(4.35-4.59)$ & $15.83(15.36-16.30)$ \\
$70-79$ & 113 & $23.26(22.96-23.57)$ & $3.02(2.93-3.10)$ & $4.55(4.55-4.66)$ & $15.59(15.31-15.88)$ \\
$80+$ & 51 & $22.87(22.71-23.04)$ & $2.90(2.79-3.00)$ & $4.71(4.56-4.87)$ & $15.25(15.06-15.45)$ \\
Total & 430 & $23.44(23.27-23.60)$ & $3.12(3.07-3.16)$ & $4.47(4.41-4.52)$ & $15.81(15.66-15.97)$ \\
\hline
\end{tabular}

El análisis estadístico se realizó utilizando un paquete de software estadístico disponible comercialmente SPSS (Statistical Package for the Social Sciences) versión 19, Chicago, Illinois, EE.UU.

El estudio fue revisado y aprobado por el Comité de Ética del Instituto de Oftalmología Conde de Valenciana de la ciudad de México.

\section{Resultados}

De un total de 565 pacientes, las mediciones de LA y parámetros asociados estaban disponibles para 865 ojos (440 derechos, 405 izquierdos). De los 440 ojos derechos estudiados, 10 fueron excluidos por historia de cirugía ocular. Finalmente, un total de 430 ojos se incluyeron en este estudio. La edad media de los pacientes fue de $70.03 \pm 10.50$ años (mediana 70 años, rango 40-96 años).

Los resultados en términos de media e IC $95 \%$ de la media de LA, PCA, GC y PCV estudiados por género y edad, la media $\pm 2 D E$ de estas variables por edad y género, y los percentiles $5,10,25,50,75,90$ y 95 de estas variables se resumen en las tablas 1, 2 y 3, respectivamente. La figura 1 muestra el histograma de la distribución de LA, PCA, GC y PCV. La tabla 3 resume la asimetría y curtosis de las variables.

La LA media fue de $23.44 \pm 1.71 \mathrm{~mm}$ (mediana: 23.15; rango: 20.48-35.05 mm; IC 95\%: 23.27-23.60). La PCA media fue de $3.12 \pm 0.46 \mathrm{~mm}$ (mediana: $3.13 \mathrm{~mm}$; rango: $1.77-4.54 \mathrm{~mm}$; IC 95\%: 3.07-3.16). El GC medio fue de $4.47 \pm 0.57 \mathrm{~mm}$ (mediana: $4.50 \mathrm{~mm}$; rango: 3.18-5.78; IC 95\%: 4.41-4.52). La PCV media fue de $15.81 \pm 1.63 \mathrm{~mm}$ (mediana: $15.58 \mathrm{~mm}$; rango: 12.98 26.78; IC 95\%: 15.66-15.97).

Las variaciones por género estuvieron presentes en todos los parámetros evaluados. Las mujeres
Tabla 2. Rango ( \pm 2 desviaciones estándar) de longitud axial (LA), profundidad de cámara anterior (PCA), grosor del cristalino (GC) y profundidad de cámara vítrea (PCV) según sexo y edad

\begin{tabular}{|c|c|c|c|c|}
\hline $\begin{array}{c}\text { Edad } \\
\text { (años) }\end{array}$ & LA (mm) & PCA $(\mathrm{mm})$ & GC $(\mathrm{mm})$ & PCV $(\mathrm{mm})$ \\
\hline $\begin{array}{c}\text { Varones } \\
40-49\end{array}$ & $22.73-23.38$ & $2.89-3.88$ & $3.66-4.61$ & $15.23-15.67$ \\
$50-59$ & $22.69-25.23$ & $2.85-4.07$ & $3.41-4.97$ & $15.49-18.00$ \\
$60-69$ & $21.17-29.56$ & $2.32-4.54$ & $3.46-5.66$ & $13.42-21.19$ \\
$70-79$ & $22.31-28.15$ & $1.77-4.11$ & $3.23-5.38$ & $14.65-20.72$ \\
$80+$ & $21.38-25.42$ & $2.40-3.97$ & $3.40-5.51$ & $13.77-17.53$ \\
\hline & & & & \\
\hline Mujeres & & & & \\
$40-49$ & $21.04-28.45$ & $2.46-4.34$ & $3.20-5.10$ & $13.57-20.51$ \\
$50-59$ & $20.81-33.52$ & $2.12-4.26$ & $3.18-5.43$ & $13.51-25.35$ \\
$60-69$ & $20.48-35.05$ & $2.24-4.12$ & $3.28-5.78$ & $12.98-26.78$ \\
$70-79$ & $21.11-31.51$ & $2.04-4.07$ & $3.33-5.74$ & $13.22-22-29$ \\
$80+$ & $21.84-24.75$ & $2.40-3.97$ & $3.54-5.73$ & $13.57-17.48$ \\
\hline
\end{tabular}

presentaron una menor LA, explicada principalmente por una menor PCA y una menor PCV. Sin embargo, mostraron un GC algo mayor.

Las personas de 40 a 49 años de edad, comparadas con personas mayores de 80 años, mostraron una mayor LA (diferencia media, $0.72 \mathrm{~mm}$ ), una mayor PCA (diferencia media, $0.52 \mathrm{~mm}$ ) y una mayor PCV (diferencia media, $0.82 \mathrm{~mm}$ ). Sin embargo, personas mayores de 80 años presentaron un mayor GC (diferencia media, $0.62 \mathrm{~mm}$ ).

Entre las personas de 50 a 59 años, en promedio, se observó una menor LA (diferencia media, $0.20 \mathrm{~mm}$ ) comparada con personas una década más jóvenes. Las personas mayores mostraron una PCA menor (0.26 mm) y una PCV menor (0.09 mm), pero un GC mayor $(0.14 \mathrm{~mm})$.

Cuando se analizó por décadas, los parámetros de LA, PCA y PCV disminuyeron a expensas de un aumento del GC. 
Tabla 3. Percentiles, asimetría, curtosis y rango intercuartil de longitud axial, profundidad de cámara anterior, grosor del cristalino grosor del cristalino

\begin{tabular}{|c|c|c|c|c|c|c|c|c|c|c|}
\hline & \multicolumn{7}{|c|}{ Percentiles } & \multicolumn{3}{|c|}{ Índices normales de distribución } \\
\hline & $5 \%$ & $10 \%$ & $25 \%$ & $50 \%$ & $75 \%$ & $90 \%$ & $95 \%$ & Asimetría & Curtosis & Rango intercuartil \\
\hline Longitud axial & 21.68 & 21.99 & 22.59 & 23.15 & 23.85 & 24.63 & 26.53 & 2.99 & 12.81 & 1.26 \\
\hline $\begin{array}{l}\text { Profundidad de } \\
\text { cámara anterior }\end{array}$ & 2.38 & 2.47 & 2.79 & 3.13 & 3.44 & 3.70 & 3.93 & 0.06 & -0.40 & 0.65 \\
\hline Grosor cristaliniano & 3.49 & 3.64 & 4.08 & 4.50 & 4.89 & 5.23 & 5.44 & -0.08 & -0.58 & 0.80 \\
\hline $\begin{array}{l}\text { Profundidad de } \\
\text { cámara vítrea }\end{array}$ & 13.99 & 14.42 & 15.01 & 15.58 & 16.25 & 16.90 & 18.31 & 2.90 & 12.41 & 1.24 \\
\hline
\end{tabular}
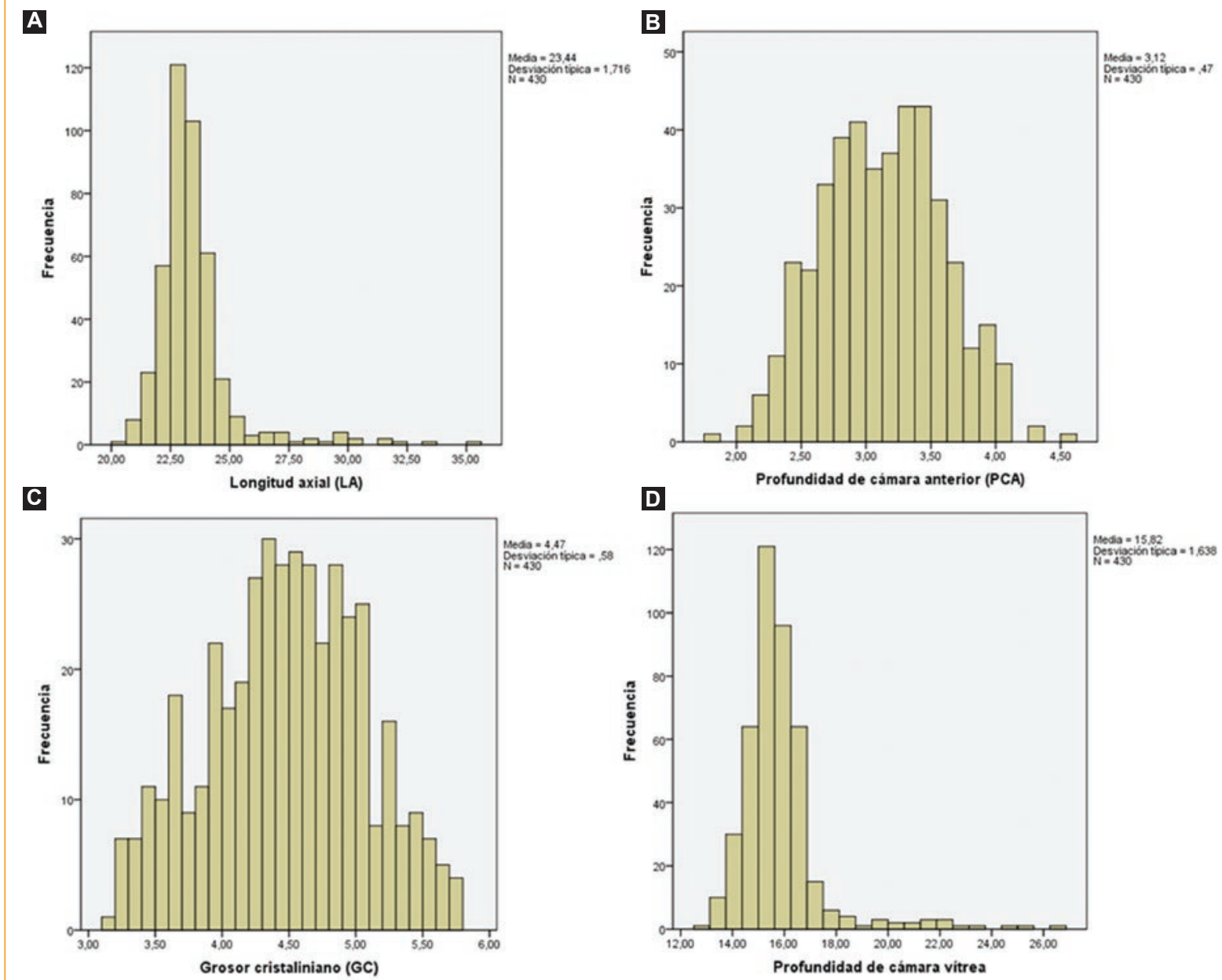

Figura 1. Distribución de longitud axial (A), profundidad de cámara anterior (B), grosor del cristalino (C), grosor del cristalino (D).

\section{Discusión}

Nuestro estudio provee información sobre los componentes biométricos oculares en adultos mexicanos mayores de 40 años que viven en la Ciudad de México $y$ alrededores.
La LA promedio en la población mexicana estudiada $(23.44 \mathrm{~mm} \pm 1.71 \mathrm{~mm})$ fue mayor a la reportada previamente en una población china adulta (The Tanjong Pagar Survey: LA $23.23 \pm 1.17 \mathrm{~mm})^{8}$ y mayor a la encontrada población adulta india (The Central India Eye and Medical Study: LA $22.6 \pm 0.91 \mathrm{~mm})^{12,13}$. En una población 
iraní, Hashemi, et al. ${ }^{14}$ reportaron una LA promedio de $23.14 \mathrm{~mm}$. Al analizar los demás factores también encontramos valores promedio menores respecto de la población estudiada. Sin embargo, todos estos estudios fueron realizados en poblaciones asiáticas.

El estudio LALES (Los Angeles Latino Eye Study) ${ }^{15}$ incluyó 5,588 latinos mayores de 40 años y el valor de la LA promedio fue de $23.38 \pm 1.01 \mathrm{~mm}$, un valor muy similar al de nuestra población. Estos resultados avalan la suposición sobre la importancia étnica en los valores biométricos.

Villanueva-Pérez, et al. (2009) ${ }^{16}$, en un estudio comparativo en población mexicana sobre la PCA en 26 pacientes, encontraron que la PCA promedio con biómetro ultrasónico modo A por inmersión era de $3.29 \pm 0.37 \mathrm{~mm}$.

Encontramos que las personas mayores tienen una menor LA, una PCA más estrecha, una PCV menor, pero un mayor GC. Estos datos biométricos coinciden con patrones de medidas observados en estudios precedentes en diferentes poblaciones ${ }^{7,9}$. Si bien un incremento en la LA es esperable desde la infancia hasta la adolescencia, es difícil de explicar el acortamiento del ojo desde la mediana edad. Una de las desventajas de nuestro estudio ha sido no comparar estas variaciones en el tamaño del globo ocular a través del tiempo. Sin embargo, Gudmundsdottir ${ }^{17}$ et al. demostraron, en un estudio de cohorte de 5 años, que esto podría deberse a muchas causas desconocidas, entre ellas, la atrofia ocular.

Una disminución de la PCA y un incremento del GC con la edad es otro de los hallazgos en este y otros estudios. El incremento en el GC con la edad se atribuye a la aposición regular de fibras del cristalino durante toda la vida, lo que causa una disminución de la $\mathrm{PCA}^{18}$.

Todos los datos biométricos analizados en este estudio han sido mayores en hombres. Todos los estudios mostraron valores de LA y PCA mayores en hombres, pero los resultados entre GC y género han sido contradictorios.

\section{Conclusión}

La LA y sus factores asociados (PCA, GC y PCV), comparados con otros estudios realizados en poblaciones asiáticas y americanas, mostraron valores medios. Excepto el GC, que aumenta con la edad, el resto de los valores decrecen con la edad. Además, todos los valores biométricos fueron más altos en hombres que en mujeres.

\section{Conflicto de intereses}

Los autores declaran no tener ningún conflicto de intereses.

\section{Responsabilidades éticas}

Protección de personas y animales. Los autores declaran que para esta investigación no se han realizado experimentos en seres humanos ni en animales.

Confidencialidad de los datos. Los autores declaran que han seguido los protocolos de su centro de trabajo sobre la publicación de datos de pacientes.

Derecho a la privacidad y consentimiento informado. Los autores han obtenido el consentimiento informado de los pacientes y/o sujetos referidos en el artículo. Este documento obra en poder del autor de correspondencia.

\section{Bibliografía}

1. Verhulst E, Vrijghem JC. Accuracy of intraocular lens power calculations using the Zeiss IOL master. A prospective study. Bull Soc Belge Ophtalmol. 2001;281:61-5.

2. Saka N, Ohno-Matsui K, Shimada N, Sueyoshi S, Nagaoka N, Hayashi W, et al. Long-term changes in axial length in adult eyes with pathologic myopia. Am J Ophthalmol. 2010;150:562-8.

3. Ruiz-Moreno JM, Montero JA, de la Vega C, Alio JL, Zapater P. Retinal detachment in myopic eyes after phakic intraocular lens implantation. J Refract Surg. 2006;22:247-52.

4. Hosny M, Alió JL, Claramonte P, Attia WH, Pérez-Santonja JJ. Relationship between anterior chamber depth, refractive state, corneal diameter and axial length. J Refractive Surgery. 2000;16(3):336-40.

5. Wang F, Zhou XD, Zhou SZ. Study of the relation between ocular axial biometry and refraction. Zhonghua Yan Ke Za Zhi. 1994;30(1):39-40.

6. Shih YF, Chiang TH, Lin LL. Lens thickness changes among schoolchildren in Taiwan. Invest Ophthalmol Vis Sci. 2009:50:2637-44.

7. Warrier S, Wu HM, Newland HS, Muecke J, Selva D, Aung T, et al. Ocular biometry and determinants of refractive error in rural Myanmar: the Meiktila Eye Study. Br J Ophthalmol. 2008;92:1591-4

8. Wong TY, Foster PJ, Ng TP, Tielsch JM, Johnson GJ, Seah SK. Variations in ocular biometry in an adult Chinese population in Singapore: the Tanjong Pagar Survey. Invest Ophthalmol Vis Sci. 2001;42:73-80.

9. He M, Huang W, Li Y, Zheng Y, Yin Q, Foster PJ. Refractive error and biometry in older Chinese adults: the Liwan eye study. Invest Ophthalmol Vis Sci. 2009;50:5130-6.

10. Hoffer KJ. Axial dimension of the human cataractous lens. Arch Ophthalmol. 1993;111:914.

11. Hoffer KJ. Biometry of 7500 cataractous eyes. Am J Ophthalmol. 1981;99:360.

12. Jonas JB, Nangia V, Gupta R, Khare A, Sinha A, Agarwal S, et al. Anterior chamber depth and its associations with ocular and general parameters. The Central India Eye and Medical Study. Clin Experiment Ophthalmol. 2012;40:550-6.

13. Jonas JB, Nangia V, Gupta R, Sinha A, Bhate K. Lens thickness and associated factors. The Central India Eye and Medical Study. Clin Experiment Ophthalmol. 2012;40:583-90.

14. Hashemi H, Khabazhoob M, Miraftab M, Emamian MH, Shariati M, Abdolahinia $\mathrm{T}$, et al. The distribution of axial length, anterior chamber depth, lens thickness, and vitreous chamber depth in an adult population of Shahroud, Iran. BMC Ophthalmol. 2012;12:50.

15. Shufelt C, Fraser-Bell S, Ying-Lai M, Torres M, Varma R. Los Angeles Latino Eye Study Group. Refractive error, ocular biometry, and lens opalescence in an adult population: The Los Angeles Latino Eye Study. Invest Ophthalmol Vis Sci. 2005:46:4450-60.

16. Villanueva G, Perdiz L. Profundidad de la cámara anterior mediante inmersión, Orbscanll, OCT Visante y UBM. Estudio comparativo. Rev Mex Oftalmol. 2009;83(4):221-5.

17. Gudmundsdottir $E$, Arnarsson A, Jonasson F. Five-year refractive changes in an adult population: Reykjavik Eye Study. Ophthalmology. 2005;112:672-7.

18. Bron AJ, Vrensen GFJM, Koretz JF, Maraini G, Harding JJ. The ageing lens. Ophthalmologica. 2000;214:86-104. 